\title{
Progress in Gene Therapy for Chronic Heart Failure
}

\author{
Zhi-qiang Yin, MD, ${ }^{1}$ Wan-hong Xing, $\mathrm{MD}^{2}$ \\ ${ }^{1}$ Shanxi Medical University, P.R.China; ${ }^{2}$ Department of Cardiothoracic Surgery, Nanchong Central Hospital/The Second Clinical \\ College of North Sichuan Medical College, P.R.China
}

\section{ABSTRACT}

Chronic heart failure (CHF) is still the leading cause of morbidity and mortality worldwide, and carries with it large economic and social burdens. Although steady and substantial progress has been made in reducing mortality from heart failure using conventional treatments, novel pharmacologic and surgical interventions have not been effective in extending five year survival rates. Therefore, it is necessary to explore new therapies. Gene therapy was introduced in 1970s with the development of recombinant DNA technology. Due to recent progress in the understanding of myocardial metabolism and application of vector based gene transfer strategies in animal models and initial clinical trials, gene therapy possibly affords an ideal treatment alternative for CHF. In last 2 decades, much research has been done on gene therapy, using various genes, signal transduction passages and delivery methods to treat advanced heart failure. Current research in ischemic heart disease (IHD) mainly focuses on stimulating angiogenesis, modifying the coronary vascular environment, and improving the vascular endothelial function with localized gene coated catheters and stents. Compared with standard ischemic heart disease treatment, the main goal of gene therapy for $\mathrm{CHF}$ is to inhibit apoptosis, reduce the undesirable remodeling and increase contractility through the most efficient cardiomyocyte transfection [Katz 2012a]. In this paper, we review various gene transfer technologies in ischemic heart disease and heart failure models, and discuss the advantages and disadvantages of these strategies in vector-mediated cardiac gene delivery, with the main focus on the high efficiency approach of a molecular cardiac surgery delivery system.

\section{INTRODUCTION}

At present, chronic heart failure $(\mathrm{CHF})$, induced by ischemic heart disease (IHD), cardiomyopathy, and congenital heart disease, among others, is still one of the main causes of mortality globally. Chronic cardiovascular disease is a major cause of mortality and morbidity in developed countries. About 85 million Americans suffer from acute and chronic cardiovascular disease (CVD), with mortality rates accounting

Received November 2, 2017; accepted December 30, 2017.

Correspondence: Wan-hong Xing,MD, Department of Cardiothoracic Surgery, Nanchong Central Hospital/The Second Clinical College of North Sichuan Medical College, Nanchong 637000, P.R.China; (e-mail:xwhcardia@sina.com). for more than $33 \%$ of all deaths in 2010, 4.4 times more than the morbidity of cancer. Direct and indirect costs resulting from CVD in the USA amount to $\$ 315.4$ billion. Coronary artery disease $(\mathrm{CAD})$ is the primary risk factor and immediate cause of CVD, and its progression is usually the result of a single or successive myocardial infarction events, with final progression to CHF. As a result, 22 million Americans are diagnosed with $\mathrm{CHF}$ each year with up to $50 \%$ mortality expected within the next 5 years; the annual costs alone exceed $\$ 34$ billion. But up to now, heart failure is still an inextricably difficult problem. There are several main treatments for heart failure, including multidrug treatment programs, biventricular pacing, mechanical cardiac support, such as left ventricular assist devices, cardiac transplant, and gene therapy. Although medical treatment with cardiotonic agents, diuretics, $\beta$-blockers, angiotensin-converting enzyme inhibitors, and other afterload reduction agents have provided symptomatic relief and survival benefits, the survival of the most advanced heart failure patients is low with medical management alone [Packer 2001; Rose 2001]. Cardiac transplant is an ideal treatment for end-stage heart failure patients. However, more than $35 \%$ of patients die in the course of waiting for the donor heart due to the limited donor pool. In addition, older patients (usually $\geq 65$ years) are unlikely to be considered suitable candidates for heart transplantation [Hou 2012]. Ventricular assist device (VAD) technology is mainly applied as a transitional treatment of end-stage heart failure before heart transplantation, but can also be used as an alternative to heart transplantation in selected patients [Hou 2012; Wever-Pinzon 2012]. VADs can also cause serious complications, such as hypoxemia, infection, sepsis, hemorrhage, embolism and mechanical failure. Cerebral thrombosis and cerebral embolism can occur in 3\%-47\% patients with LVADs.

With advances in cardiovascular research, including cardiac arrhythmias, heart failure, and ischemic heart disease, vector-based gene transfer strategies in animal models and in initial clinical trials have demonstrated possible viable alternatives for the treatment of heart failure. Extensive preclinical studies have provided solid data indicating a clinical potential for gene therapy. Selected transgenic expression in the myocardium increases contractility, restores global function, and completely reverses chronic heart failure in some cases [Rengo 2009; Fargnoli 2011; Katz 2011; Rengo 2011]. However, this area of research is still in its infancy, and has many unresolved problems which limit the clinical application of gene therapy.

Gene therapy is a complex process in many aspects, such as the targeted tissue, delivery pathway, cell transport, gene 
expression regulation, biological activity and dose of therapeutic transgene [Katz 2014c]. Inadequate delivery and transfection efficiency has been one of the main limitations of gene therapy. This efficiency can be improved by developing better gene delivery methods and vectors. So far, there has not been a delivery system or a virus serotype optimized for cardiac orientation without collateral expression in other tissues. A large number of gene transfer techniques have been studied in cardiovascular gene therapy. These findings indicate that the physical path of gene delivery is even more important than the vector system, and the target of gene delivery should be minimum or zero expression of the accessory organs [Lin 1990; Guzman 1993; Barr 1994; French 1994; Katz 2014b]. In recent years, Bridges and colleagues have established and done research on a molecular cardiac surgery delivery system for gene therapy. The results have shown even distribution of genes in the myocardium, high transgene efficiency and little collateral infection. This review will detail the present status of a gene delivery system for IHD and CHF, with a focus on molecular cardiac surgery as a promising alternative in the future to treat chronic heart failure.

\section{METHODS}

In general, a common goal of gene therapy for ischemic heart disease and heart failure is to correct the molecular mechanisms in the coronary vessels and myocardium to reverse cardiac damage [Katz 2012a]. In fact, the methods of gene delivery are almost the same, except for the widespread application of intracoronary wall delivery in CAD. Up to now, there have been various gene delivery strategies for gene transfer to the myocardium, which can be divided into the site of injection, the interventional approach and variations in the cardiac circulation system [Katz 2011].

In this section we will briefly discuss:

- Direct myocardial delivery

- The percutaneous catheter-based gene delivery system

- The V-focus system

- The surgical delivery strategy

\section{Direct myocardial delivery and other mechanical and physi- cal approaches}

Direct myocardial gene delivery is attractive since it can target specific cardiac regions, and achieve high concentrations of local vector. However, these methods may lead to systemic exposure through unintentional myocardial vascular leakage and spillage outside of interstitial space [Katz 2013b] .

Site of injection approach. Direct injection of vector into the myocardium can be achieved by a surgical or percutaneous approach. It overcomes the common disadvantages of vascular pathways, including the first-pass effect of liver and spleen, the role of neutralizing antibodies, the $\mathrm{T}$-cell response and the permeability of the endothelial barrier [Kawase 2011]. Several groups have demonstrated the feasibility of direct injection of genes into the myocardium. Surgical gene transfer can be implemented through a subxyphoid or transthoracic approach to specific myocardial sites to achieve a high localized vector concentration, and it is easy to implement in cardiothoracic surgery (Figure 1A). The application of this approach has resulted in successful treatment of angiogenesis [Schwarz 2000] and focal arrhythmias therapy through effects on cellular electrophysiology [Edelberg 2001; Katz 2014b]. However, direct injection into the myocardium can only result in gene expression over a limited region due to the lack of carrier diffusion. Therefore, the method is not sufficient enough to achieve global distribution. Furthermore, needle injection also causes secondary acute inflammation and innate immune response [Katz 2014b]. In addition, this method does not rule out the system vector diffusion and collateral organ exposure. Because of low transfection efficiency and other shortcomings, the needle injection is not an appropriate therapeutic approach. This approach may be applicable for regional strategies.

Image-guided injections featuring cardiac mapping technology. Catheter based needle local injection can be achieved using various types of catheters as well as a variety of guided modes, including fluoroscopy, electromagnetism and $3 \mathrm{D}$ mapping systems (Figure 1B). Percutaneous gene delivery allows the use of controllable needle-tip catheters to inject the
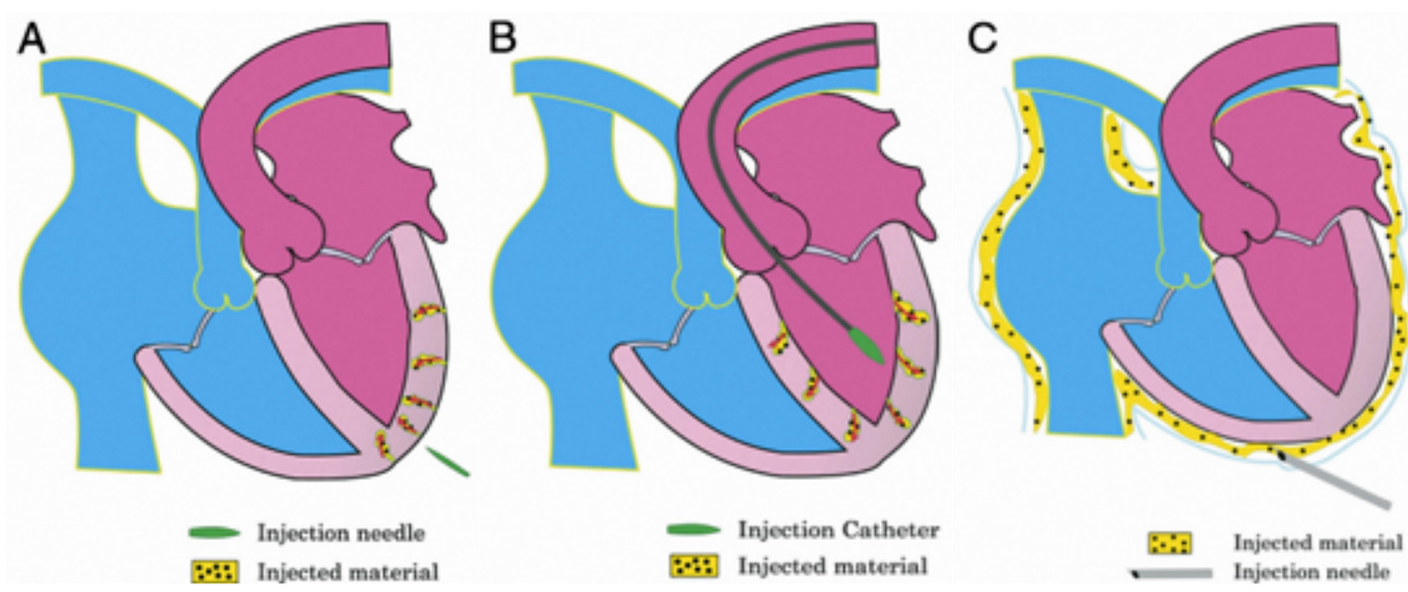

Figure.1 Ventricular injections of vectors. A. Direct surgical injections; B. Catheter based intracardiac injections; C. pericardial delivery. 
vector into the predetermined regions of endocardium. The catheter tip can be guided by fluoroscopy and electromechanical mapping. The non-fluoroscopic electro-mechanical mapping system is even more suitable for the endocardial gene transfer approach. Various specialized catheters have been developed to aid in direct gene delivery, such as the NOGA catheter. The NOGA is a modified version of clinical cardiac imaging modalities, including echocardiography and magnetic resonance imaging (MRI). Echocardiography can not only provide the assessment of regional wall motion, valvular regurgitation, pericardial effusion, but also provides imaging of cardiac structure. Another major advantage of echocardiography is the ability to detect the leakage of the vector in real time, which is not possible with fluoroscopy. Intracardiac echocardiography can predict the injection site accurately and achieved gene expression in $95 \%$ of injection sites in a porcine model. The relative weak point of the echocardiography is limited to a single planar image and requires fluoroscopy to guide the catheter. $3 \mathrm{D}$ echocardiography, which is now widely used in clinical practice, provides real-time feedback on the localization and retention of injectate and is a valuable method to enhance gene transfer. Cardiovascular MRI can offer the ability to optimize delivery, and can easily delineate the catheter position in relation to endocardial/epicardial surfaces and also distinguish between papillary and valvular structures. The main shortcomings of MRI guidance are long surgical time, high cost, and limited application in patients with implanted various cardiac devices [Katz 2013b].

Pericardial delivery. An alternative site of injection for gene delivery is intra-pericardial (Figure 1C). Intra-pericardial injection can be injected through the endocardium or epicardium. Because the pericardial cavity around the heart also secretes vasoactive substances, it is speculated that injection of the virus within the pericardium may spread through the barrier to the myocardium and coronary arteries. It is known that the pericardial envelope can be easily accessed. In addition, since the pericardium is a closed cavity, it can be used to prolong the contact time with genetic vectors. It has been shown that intra-pericardial injection is an efficient and safe method to deliver a transgene to the myocardium. Although some researchers have demonstrated that pretreatment before adenovirus administration have increased transgene activity [Fromes 1999; Lamping 1997; Roques 2007], the disadvantage of this approach is that the pericardial envelope seems to be relatively tight, so diffusion of genes across this membrane is difficult. In addition, the most common and severe complication of pericardiocentesis in clinical as well as in large animal research is hydropericardium, and the rapid accumulation of air and fluid may be fatal [Ladage 2012]. The method may not be applicable to patients with adhesions after multiple procedures [Kornowski 2000]. Although animal studies have shown the feasibility and safety of the intrapericardial delivery approach, considering the various factors above, pericardial delivery is unlikely to be particularly promising in clinical applications.

Physical methods that improve permeability of cell membranes, and other mechanical methods. In many aspects, the efficiency of electroporation, which refers to the application of short duration, high intensity electric pulses which can enhance the cardiomyocyte membrane permeability and increase DNA uptake, is relatively high [Katz 2013a; Katz 2013b; Katz 2014b]. At present, it is the most commonly used physical method, but the high voltage application may affect the stability of genomic DNA and transgene expression in the tissue with uneven distribution [McMahon 2004; Katz 2013a]. Sonoporation is another commonly used physical method, in which the DNA is in micro-bubbles filled with gas then destroyed by ultrasonic pulses. This method can
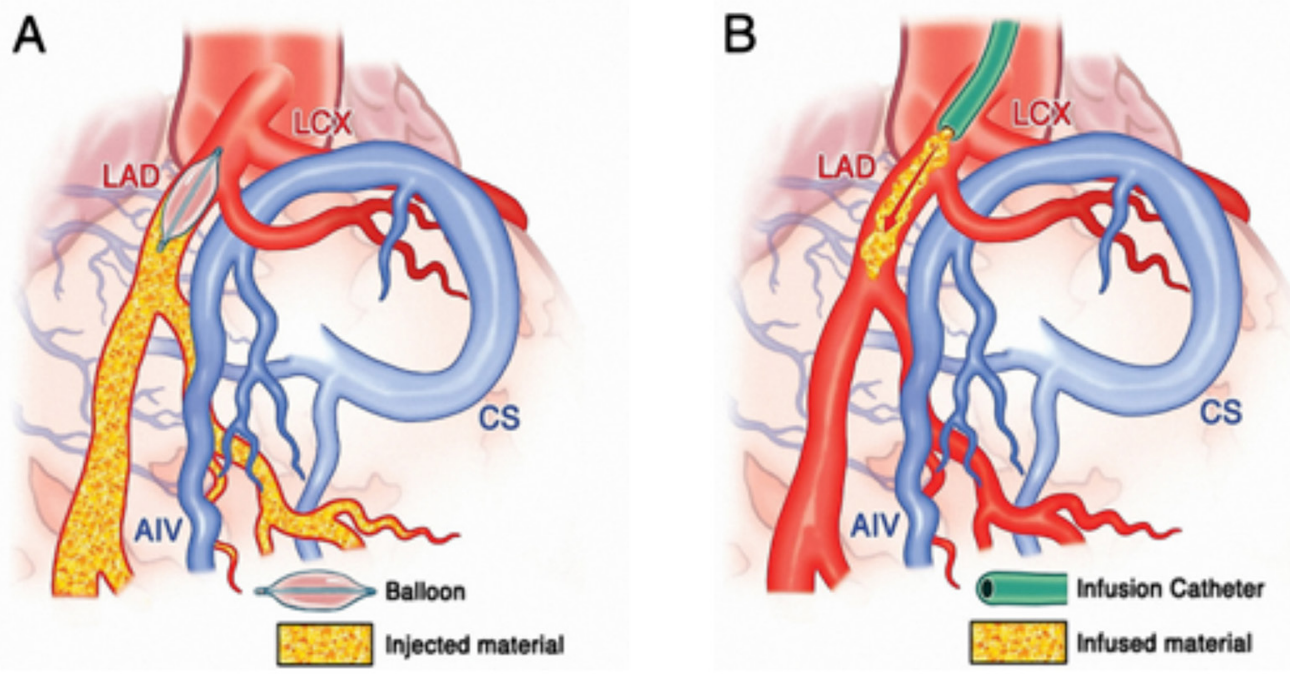

Figure 2. Antegrade injections of vectors through coronary vessels. A. Antegrade injection while blocking the coronary artery; B. antegrade injection while not blocking the coronary artery. LAD, left anterior descending coronary artery; AIV, anterior interventricular coronary vein; LCX, left circumflex coronary artery; CS, coronary sinus 
improve the permeability of cell membrane by ultrasound. Transfection efficiency was determined by acoustic pressure, pulse duration and time of cells exposure to ultrasound. Some other frequently used physical methods include microinjection, laser irradiation and magnetic field transfection. Microinjection directly delivers DNA into the nucleus, resulting in a higher level of gene expression than standard injection. Although there is such an advantage, this method is impractical with in vivo cardiac applications because of the scale factor. The thermal effect of the laser beam can increase the permeability of cell membrane to exogenous DNA. This technique has not yet been extensively studied, and the types and extent of damage caused by laser irradiation still remains unknown. Magnetofection refers to the incorporation of DNA nanoparticles into the heart under the action of strong magnetic field with higher transfection efficiency. However, the equipment required for this technique is very expensive. Other frequently mechanical methods include liquid jet injection and particle bombardment. Liquid jet injection uses a high pressure gas injection through a sharp nozzle to treat a molecular liquid. The impact force generated by the jet can perforate the cell membrane [Katz 2013a; Katz 2013b, Fargnoli 2014; Katz 2014b]. The results showed that the transfection efficiency was 50-fold higher than with conventional needle injection, but less than that of particle bombardment [Katz 2014b]. Particle bombardment technology is based on the use of pressurized medical gases to accelerate the high-speed metal-DNA complex, which is covered with pDNA micrometer sized heavy metal particles. This transfer is achieved by direct penetration of the plasma membrane to the target cells. As a result of gene transfer by needle injection, and the existence of isletlike vector clusters with the host inflammatory cells gathered around the injection site, some researchers believe that liquid jet injection and particle bombardment can help to solve these problems [Katz 2013b]. However, the main limitation of particle bombardment is that only the surface layer of myocardium is transfected [Fargnoli 2017].

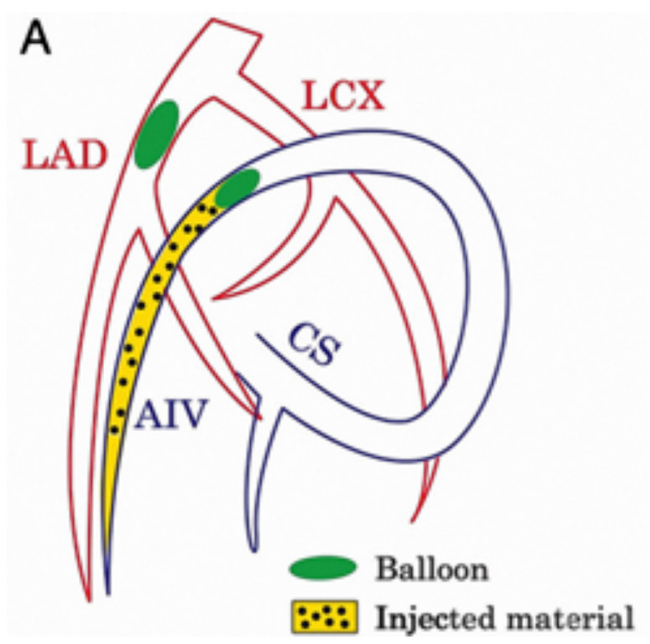

Percutaneous catheter-based intracoronary gene delivery system

Since most heart diseases have diffuse heart tissue involvement, gene therapy requires global transduction into the myocardium, which can only be achieved by intravascular access. However, the effect of a simple intravenous gene delivery would be poor, probably because of first-pass pulmonary uptake and systemic spillage [Lazarous 1997; Katz 2014a]. Currently the main efforts of researchers have focused on intracoronary transfer. Percutaneous catheter-based gene delivery is simple, reliable, safe and allows vector localization; percutaneous catheter coronary injection is one of the most attractive approaches. Intracoronary gene delivery gives better access to all cardiac structures compared with other delivery methods. This technique is less invasive and it is possible to transduce genes into the target region. Some pathways, such as high-pressure gene delivery [Hajjar 1998; Wright 2001], increases gene administration up to 10 minutes [Kawase 2008] and the flow rate of infusion [Emani 2003] has been used to improve transduction efficiency. Furthermore, numerous agents that increase the permeability of the vascular bed, such as nitroglycerin, nitroprusside, serotonin, bradykinin, histamine, substance $\mathrm{P}$ and vascular endothelial growth factor (VEGF) [Kawase 2011; Ladage 2012] have been used in preclinical trials. However, clinically, these agents must be used with caution so as not to decrease systemic blood pressure.

Antegrade coronary injection. Antegrade injection into the coronary circulation, which simulates physiological coronary flow, is characterized by its capacity to deliver the vectors to the myocardium the most homogenously [Ladage 2012] and the possibility of delivery of repeated vectors. This method is also relatively easy and requires only conventional techniques of percutaneous transluminal angioplasty with commercially available catheters. This method has also been used in the successful first cardiac gene therapy trial (CUPID) [Lazarous 1997].

A. Antegrade injection by blocking the coronary artery (Figure 2A).

This process involves expanding the balloon to occlude the coronary flow, while injecting downstream of the balloon,

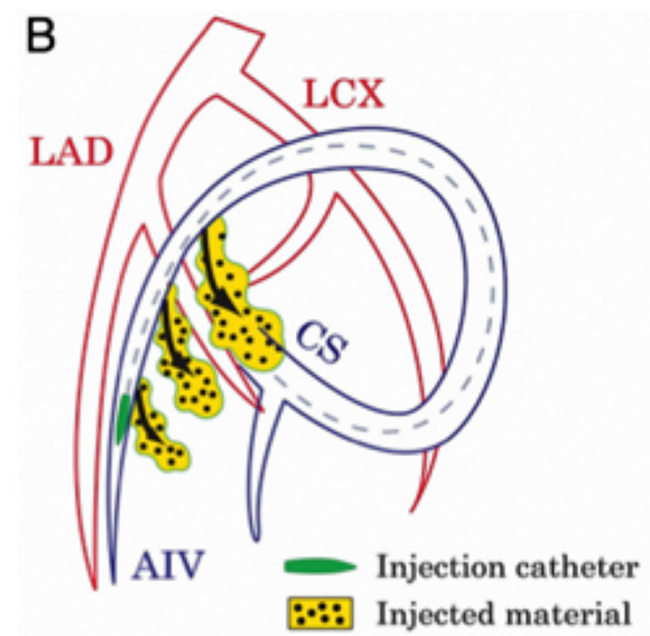

Figure 3. Retrograde injections of Vectors through Coronary Vessels. A. Retrograde infusion through the coronary sinus, while the coronary artery is blocked; B. Intramyocardial injection from a catheter within the coronary vein. 
thereby avoiding the dilution of the viral vector. However it may be not well tolerated to block antegrade flow even for a short period of time.

B. Prolonged antegrade injection without blocking the coronary arteries (Figure 2B).

This simple but effective method, which does not involve flow obstruction, can be the best method for patients with heart failure who cannot tolerate coronary artery blockade. Although this method follows the normal pattern of coronary flow, and has been shown to improve ventricular function in a large animal model of heart failure, it can only infect approximately $60 \%$ of myocytes [Kawase 2008; Kawase 2011].

A number of studies have shown that antegrade intracoronary injection only achieves a low transfection efficacy [Kawase 2008; Ladage 2012]. Simple antegrade intracoronary injection can result in more than $99 \%$ of the vectors disappearing into the systemic circulation, which lead to significant collateral organ uptake and influences the efficiency of intracoronary transfer [Katz 2014a].

Retrograde coronary injection. Retrograde injection can be achieved through the coronary sinus with the coronary artery blocked (Figure $3 \mathrm{~A}$ ) or a through a transcatheter device within a coronary vein (Figure $3 \mathrm{~B}$ ). In most cases, there are no lesions in coronary veins, so it is very attractive to use coronary veins to deliver genes, especially in patients with coronary artery disease [Raake 2008; Kawase 2011; Katz 2012a]. Second, pressure regulated retrograde perfusion into the anterior cardiac vein through the coronary sinus substantially increases gene expression in the target region due to an about ten fold increase in coronary passage time compared with other methods. Other advantages include an increase the capillary filtration ratio in the venous part of the capillary bed and overcoming the resistance of precapillary sphincters located before arterial capillaries. Furthermore, it can reduce myocardial reperfusion injury [Boekstegers 2000; von Degenfeld 2003; Katz 2012a; Katz 2013a; Katz 2014a, Katz 2014b]. Retrograde coronary infusion, compared with antegrade, provides a more uniform distribution in the myocardium [Katz 2012a]. However, a few studies showed that antegrade coronary injections were superior to retrograde injections. In addition, this approach may be problematic if it were achieved through the coronary sinus with the coronary arteries blocked, because some patients may not tolerate coronary occlusion [Hoshino 2006].

Intracoronary delivery has shown limited transfection because single pass vascular gene transfer leads to rapid dilution of the vector concentration in circulating blood. On the other hand, intracoronary delivery may have various results, such as systemic leakage and vector dissemination to collateral organs [Katz 2014b]. The results of the CUPID trial using an intracoronary delivery route showed that there was no significant improvement in left ventricular function [Katz 2014c].

\section{Methods mainly used in patients with CAD}

Transvascular intracoronary wall delivery. The ideal local gene therapy should be based on the minimally invasive techniques, which are characterized by vascular intervention and multiple surgical procedures. However, the application of these methods for vector-mediated gene transfer to the coronary artery may be limited by the following anatomical barriers: the tunica adventitia, external and internal elastic lamina, smooth muscle cells, and tunica intima within the endothelium [Katz 2012a]. The adventitia plays an important role in the process of remodeling and neointimal hyperplasia [Scott 1996]. The advantage of adventitial gene transfer technology is that it can prevent bloodflow interruption, endothelium disruption and vector leakage into the systemic circulation [Hiltunen 2000]. The gene transfer of the large peripheral arteries can be achieved by injecting the vector directly into the adventitia during surgery. Biodegradable adventitial collars, gels or aerosolization devices represent the latest approaches in optimizing transfer [Quarck 2004].
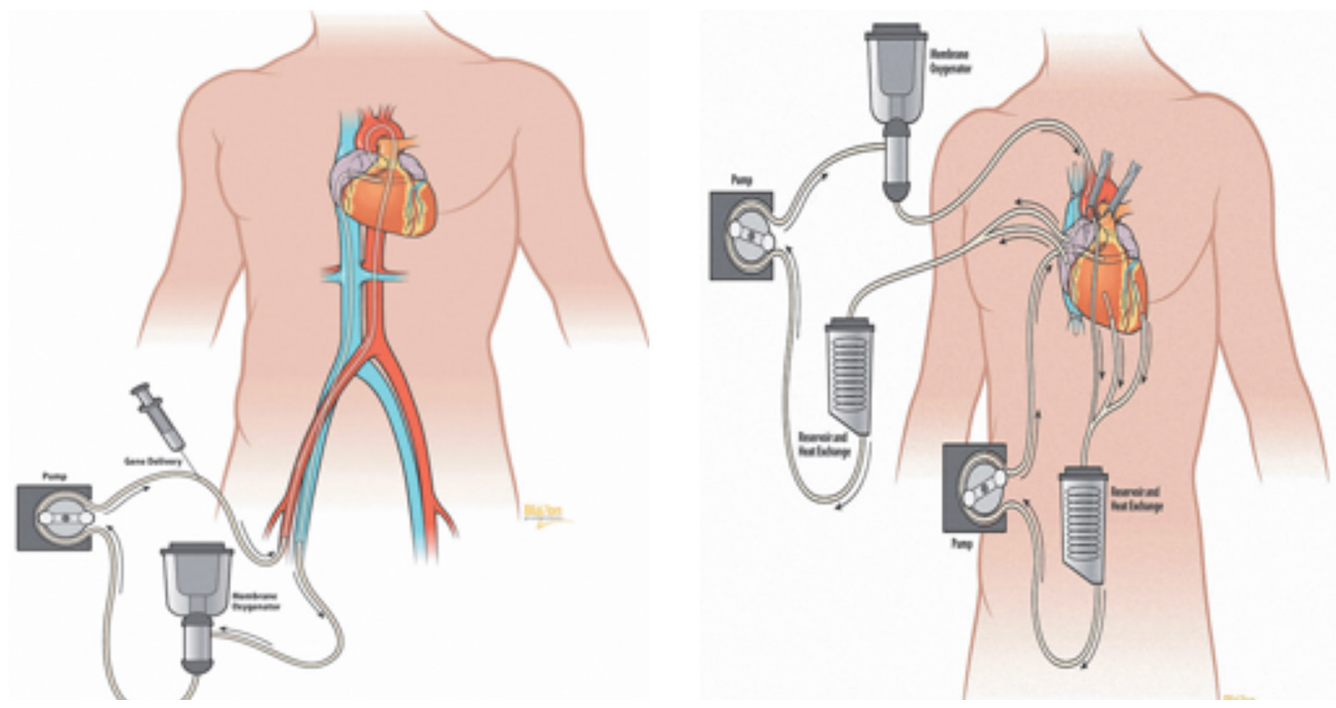

Figure 4 A. V-Focus.; Figure 4B. MCARD. Closed-loop recirculatory systems. The main differences between V-Focus and MCARD: (i) beating vs stopping heart; (ii) direction of recirculation (coronary arteries to coronary sinus vs coronary sinus to coronary arteries); (iii) time of recirculation (10 vs 20min); (iv) percutaneous catheter- based methodology vs cardiopulmonary bypass-based. 
Gene-eluting stents. Despite the successes described above, researchers now seem to prefer eluting stents. They represent the latest progress in the localized treatment of chronic coronary artery occlusive disease, re-stenosis after percutaneous transluminal coronary angioplasty, and vein graft disease after coronary artery bypass graft. [Katz 2014a]. The advantages of eluting stents include: extensive clinical experience in coronary catheterization procedures, safety, permanent scaffold structure, and acting as reservoirs for viral vectors without systemic side effects [Sharif 2004; Sharif 2006; Gaffney 2007; Fishbein 2010]. In recent years, study efforts have been focused on increasing the combination of viral or nonviral vectors in the coating stents, the elution of the target gene from the stent, and enhancing transfer to the coronary endothelium [Sharif 2006]. Further research is needed to assess the feasibility of this technique in clinical trials [Katz 2014a].

Percutaneous coronary intervention (PCI) and coronary artery bypass grafting (CABG) are mainly applied to patients suffering with coronary artery disease $(\mathrm{CAD})$ requiring revascularization. Despite the introduction of advanced new drug eluting stents and other combination products, the probability of failure of these procedures due to restenosis is still relatively high [Katz 2012a]. Some studies have estimated that more than $30 \%$ of patients with ischemic CAD may not be able to accept PCI or CABG as a result of suboptimal anatomy or the inability to perform complete revascularization [Lassaletta 2011].

\section{The V-focus system}

The V-Focus system is a minimally invasive percutaneous catheter technique for the establishment of a closed isolated regional myocardium perfusion between the coronary arteries and the coronary sinus. Coronary venous blood is drained from the coronary sinus. Following oxygenation, the blood is returned to the left main coronary artery via a roller pump. The vector is directly delivered into antegrade limb of the circuit and circulates through the myocardium for approximately 10 minutes. Research has shown an advantage in affecting the systolic and diastolic parameters of heart function after delivery of AAV2/1-mediated SERCA2a compared with the antegrade coronary artery [Kaye 2007; Byrne 2008; Kawase 2011]. However, some research results showed that use of the V-Focus system can not achieve a true closed loop system and more than $99 \%$ of the injected vector leaked into the systemic circulation [Bridges 2009] (Figure 4A).

\section{Surgical delivery strategies}

Conventional cardiac catheterization strategies are attractive due to their relative safety and controllability. An obvious drawback of intracoronary delivery is low reported transduction efficiency, with cardiac expression levels within the range of 0.03 to $<1$ GC therapeutic DNA per cell. In addition, global delivery is not generally realized and expression is usually limited to the major vascular axis [Fargnoli 2011]. Transient coronary artery occlusion and retrograde perfusion can improve the transduction efficiency compared with standard intracoronary infusion, but these methods may lead to serious security problems in heart failure patients with coronary artery disease [Kaplitt 1996; Raake 2004]. In either case, the vector in the transfer site will inevitably leak into the systemic circulation. In fact, it can be found in reviews of published available data via PCR quantitative gene transfer analysis of $>100$-fold more expression in collateral organs, such as the liver versus the heart, with intracoronary delivery [Bridges 2009; Fargnoli 2011]. Therefore, advanced interventional and surgical delivery technologies are under development to address these problems, and an ideal delivery mechanism would provide safe, homogeneous myocardial delivery with limited systemic "spillover", or collateral expression, and minimize the potential immune response against the vector capsid [Katz 2014a]. In some clinical situations, surgical gene delivery may be the most efficient strategy. However, it is considered to be the most invasive method because of the significant morbidity associated with access to the myocardium. In general, surgical gene delivery may be performed during open cardiac surgery.

Recently, a large animal research study was done by Charles $\mathrm{R}$ Bridges and colleagues. They described molecular cardiac surgery procedures with recirculating vector delivery to the ovine heart in a large animal model of post ischemic heart failure. Here we briefly discuss the main points. First, direct ligation of two of coronary arteries result in infarction. The creation of an infarct was confirmed by acute ST-elevation and surrounding tissue discoloration. The right carotid artery was cannulated for systemic perfusion. The aortic root vent, superior vena cava cannula, and retrograde cardioplegia catheters were then placed, then partial $\mathrm{CPB}$ was initiated. The inferior vena cava was cannulated, then full CPB was initiated. The right and the left azygos (hemiazygos) veins were ligated, and vent cannulas were placed into the left and right ventricles. The vents were connected to the venous limb of the cardiac venous return circuit. The arterial limb of the cardiac circuit was connected to the coronary sinus catheter. The aorta was crossclamped. Cold $\left(4^{\circ} \mathrm{C}\right)$ crystalloid cardioplegic solution was delivered antegrade. The heart was isolated by tightening the snares on the superior and inferior venae cavae and crossclamping the pulmonary artery. The virus solution was injected into the retrograde catheter and recirculated for 20 minutes. Then the coronary circuit was flushed to wash out the residual vectors. The aortic crossclamp was removed and flow restored. Rewarming was initiated. The animal was subsequently weaned completely from $\mathrm{CPB}$ and the incision was closed in standard fashion. All animal subjects received critical cardiac postoperative care and survived until being humanely killed at 8 weeks (Figure 4B) [Fargnoli 2011; Katz 2014c; Katz 2017].

The main differences between V-Focus and MCARD are: (i) a beating versus stopped heart; (ii) direction of recirculation (coronary arteries to coronary sinus vs coronary sinus to coronary arteries); (iii) time of recirculation (10 $\mathrm{min}$ vs $20 \mathrm{~min}$ ); (iv) percutaneous catheter- based methodology versus cardiopulmonary bypass-based.

Ischemic preconditioning (IP) is a very important step in the experimental procedure, which has an effect on myocardial gene transfection. Myocardial IP with a 1-min occlusion of two major coronary arteries can improve the marker gene expression. Two minutes of temporary cardiac arrest allowed the transfection of $18 \%$ cardiomyocytes, while an extension of time to 5 minutes resulted in a cardiac transfection of $43 \%$ of cells. A pig model demonstrated that retrograde delivery during $10 \mathrm{~min}$ of ischemia increased reporter gene expression compared with control, and the use of two periods of ischemia resulted in more homogeneous transmural expression. 
These and other data confirm that IP is involved in the effectiveness of gene transfer [Boekstegers 2000; Hayase 2005; Katz 2013a].

MCARD with retrograde perfusion through the coronary sinus is an especially reasonable and effective method. The coronary sinus has become a clinically important structure due to its access for different cardiac procedures. Many authors believe that coronary venous infusion prolongs adhesion time of the vector to the cardiac endothelium. This effect directly leads to an increased endothelial permeability as well as a higher pressure gradient across the interstitial capillaries and venules, which promote the transfer of macromolecular particles into the interstitium of the heart [Kupatt 2002; Giordano 2003; Boekstegers 2004]. Retrograde coronary venous delivery can preserve myocardium and deliver oxygenated blood during ischemia. It has been shown that this perfusion method can reduce myocardial reperfusion injury [Katz 2014a], accelerate the thrombolysis of coronary artery, protect the whole and local myocardial function during the open heart surgery, and limit the infarct size [Katz 2013a]. However, a main limitation of retrograde injection is the relatively poor perfusion in the right ventricle.

Bridges and colleagues presented a pharmacokinetics analysis of molecular cardiac surgery with recirculating delivery (MCARD) of scAAV6-ARKct. qPCR and Western blots were used to determine biodistribution. PK model data revealed that only $0.61 \pm 0.43 \%(\log \mathrm{GC} / \mathrm{mL})$ of the original dose remained in the blood after delivery, and complete clearance from the system was achieved at 1 week. A PK transfer function revealed a positive correlation between exposure and in vivo transduction. MCARD could provide a viable method for establishing a better relationship between vector exposure and in vivo transduction. Using this methodology, it may address a critical need to establish an effective therapeutic window [Fargnoli 2011].

The Bridges' group also investigated the effect of molecular cardiac surgery with recirculating delivery (MCARD)-mediated carboxylterminus of the $\beta$-adrenergic receptor kinase ( $\beta A R K c t)$ gene transfer on cardiac mechanoenergetics and $\beta$-adrenoreceptor ( $\beta \mathrm{AR}$ ) signaling. Mechanoenergetic studies using magnetic resonance imaging were performed at 4 and 8 weeks after MCARD separately. Tissues were analyzed with realtime quantitative polymerase chain reaction (RT-qPCR) and Western blotting. RT-qPCR confirmed robustness of $\beta$ ARKct expression throughout the left ventricle, and undetectable expression in extracardiac tissues. Quantitative Western blot data confirmed higher expression of $\beta A R K c t$ in the left ventricle: 0.46 \pm 0.05 versus 0.00 in lung and liver $(P<.05)$. Survival was $100 \%$ and laboratory parameters of major organ function were within normal limits. It showed that MCARD-mediated $\beta A R K c t$ delivery was safe, resulted in robust cardiac-specific gene expression, enhanced cardiac contractility and lusitropy, increased adrenergic reserve, and improved energy utilization efficiency in a preclinical large animal model [Katz 2012b].

The Bridges' group conducted a related experiment in 2014 , in which markers of oxidative stress were significantly reduced in the infarct zone in the MCARD group. There was no positive $T$ cell mediated immune response in the MCARD group at any time point. Myocyte hypertrophy was also significantly attenuated in the MCARD group compared to control.
Cardiac overexpression of the SERCA2a gene by MCARD is a relatively safe therapeutic intervention. It significantly improves left ventricular function, reduces the markers of oxidative stress, inhibits myocyte hypertrophy, arrests of reconstruction, and does not induce a T-cell-mediated immune response [Katz 2014c].

In summary, MCARD, the closed-loop recirculatory system, which has the most important characteristic-separation of the coronary vascular bed from the systemic, can avoid circulating blood dilution and get a $>100$-fold increase in transduction efficiency. Thus, this technology can prolong vector residence time in myocytes and has the ability to manipulate endothelial permeability. Moreover, the system has the ability to washout the vectors after gene delivery, limiting collateral organ exposure and avoiding an immune response to the vectors [White 2011; Katz 2014b]. Conversely, in the case with incomplete cardiac isolation, immunohistochemical staining revealed reporter gene expression in the lungs, liver, kidneys and spleen likely due to the relatively high systemic biodistribution of vector genomes [Katz 2010]. Furthermore, the surrounding tissue of all main organs after MCARD ${ }^{\mathrm{TM}}$ showed no evidence of significant inflammation or tissue damage [White 2011].

However, MCARD is performed through the chest with cardiopulmonary bypass, and it is not clear whether patients with advanced heart failure could tolerate such a procedure for gene transfer. First, the risk of complications caused by this operation are relatively high, with common types of sternal dehiscence, chest infection, infective endocarditis, low cardiac output syndrome, arrhythmia, etc. Moreover, because of the open chest trauma, long incision and postoperative pain, this method may not be applicable to older patients or those in poor health. Secondly, this kind of operation is more complex than the catheter interventional operation, with longer recovery times, complex postoperative care and the high cost.

\section{DISCUSSION}

At present, clinical trials of cardiovascular gene therapy have become the second major investigated application after cancer [Edelstein 2007; Giacca 2012]. Although, in fact, cardiovascular disease remains the leading cause of mortality, morbidity and health-care expenditure around the world, and there is evidence of the benefits and feasibility of cardiac gene therapy in preclinical models, the number of oncological human trials is 7.6-times higher than those of cardiovascular diseases. There is no doubt that the insufficient number of trials has led to many problems that still can not be accurately answered, and gene therapy technology lags behind. Only the identification of a clear indications for gene therapy, combined with an optimized delivery route, may contribute to new gene therapy strategies for ischemic heart disease and HF [Katz 2014a].

To date, taking into account the entire universe of gene therapy, no single gene therapy product has been approved by Food and Drug Administration (FDA) for clinical use in the United States. Randomized clinical trials have shown that the efficacy of gene therapy is not as substantial as expected from animal studies. It is believed that the disparity between the preclinical and clinical results is attributable to the very low to undetectable levels of therapeutic expression in human myocytes. Although some 
animal models may mimic human disease, and are easy to reproduce and suitable for assessment of heart function, a significant difference exists in genetic background, rate of metabolism, neurohormonal activation and basic cardiac physiology. The method of gene transfer, which could be effective in small animals but not necessarily could be extended to larger species, therefore, could not be fully transduced into myocardial cells to establish long-term clinical efficacy [Katz 2014b]. The experiments were performed with a relatively small number of animals or normal sheep. Although coronary venous and arterial anatomy of the sheep is similar to that of humans, the study with sheep cannot necessarily be extrapolated to patients [Katz 2010].

The selection of gene transfer vector, dose level and delivery system may be the key determinant of therapeutic effects. The interactions between vector delivery and uptake, delivery route means, and the host's physical limits must be considered synergistically for a successful treatment course [Katz 2013b]. Although advances in gene delivery technology are exciting, further efforts are needed to overcome the obstacles which hinder the technologies that successfully translate into clinical practice [Melo 2005]. Specifically, given that most cases of heart failure (HF) are due to coronary vascular disease, the improvements of gene therapeutics should include therapeutic angiogenesis in the ischemic myocardium, prevention of post-angioplasty and in-stent restenosis, post-coronary bypass graft failure, and correction of the molecular mechanisms of cardiac deterioration [Quarck 2004].

\section{CONCLUSION}

Optimal transfection pathways should have the characteristics of high transfection efficiency, simple operation, small trauma, high targeting of myocardial transfection and the minimum or zero expression of the accessory organs, but existing transfection pathways fail to meet all of the above requirements. Up to now, the MCARD vector-mediated gene delivery platform is the only true closed-loop recirculation, which is undoubtedly one of the most promising gene transfer systems for providing very high expression levels in the most efficient manner. Moreover, MCARD also provide a unique opportunity to limit systemic exposure, which increases the safety factor. Although the efficacy of clinical trials is not ideal as expected, the results are not insurmountable. More sophisticated studies with advanced therapies are entering phase III clinical studies. Clinically, the MCARD approach, coupled with an appropriate therapeutic transgene, may be more applicable to patients with end stage heart failure, who would undergo cardiac surgery for other reasons, such as valve repair or replacement or ventricular assist device placement. Although the procedure is currently performed using a median sternotomy, it could be readily performed through a small anterior thoracotomy using minimally invasive or robotic surgical techniques. Therefore, the MCARD vectormediated gene delivery is still the most attractive method for ischemic heart disease and heart failure.

\section{REFERENCES}

Barr E, Carroll J, Kalynych AM, et al. 1994. Efficient catheter-mediated gene transfer into the heart using replication-defective adenovirus. Gene Ther 1:51-8.

Boekstegers P, Kupatt C. 2004. Current concepts and applications of coronary venous retroinfusion. Basic Res Cardiol 99:373-81.

Boekstegers P, von Degenfeld G, Giehrl W, et al. 2000. Myocardial gene transfer by selective pressure-regulated retroinfusion of coronary veins. Gene Ther 7:232-40.

Bridges CR. 2009. 'Recirculating cardiac delivery' method of gene delivery should be called 'non-recirculating' method. Gene Ther 16:939-40.

Byrne MJ, Power JM, Preovolos A, et al. 2008. Recirculating cardiac delivery of AAV2/1SERCA2a improves myocardial function in an experimental model of heart failure in large animals. Gene Ther 15:1550-7.

Edelberg JM, Huang DT, Josephson ME, Rosenberg RD. 2001. Molecular enhancement of porcine cardiac chronotropy. Heart 86:559-62.

Edelstein ML, Abedi MR, Wixon J. 2007. Gene therapy clinical trials worldwide to 2007--an update. J Gene Med 9:833-42.

Emani SM, Shah AS, Bowman MK, et al. 2003. Catheter-based intracoronary myocardial adenoviral gene delivery: importance of intraluminal seal and infusion flow rate. Mol Ther 8:306-13.

Fargnoli AS, Katz MG, Bridges CR. 2017. A Needleless Liquid Jet Injection Delivery Approach for Cardiac Gene Therapy. Methods Mol Biol 1521:219-226.

Fargnoli AS, Katz MG, Williams RD, Margulies KB, Bridges CR. 2014. A needleless liquid jet injection delivery method for cardiac gene therapy: a comparative evaluation versus standard routes of delivery reveals enhanced therapeutic retention and cardiac specific gene expression. J Cardiovasc Transl Res 7:756-67.

Fargnoli AS, Katz MG, Yarnall C, et al. 2011. A pharmacokinetic analysis of molecular cardiac surgery with recirculation mediated delivery of betaARKct gene therapy: developing a quantitative definition of the therapeutic window. J Card Fail 17:691-9.

Fishbein I, Chorny M, Levy RJ. 2010. Site-specific gene therapy for cardiovascular disease. Curr Opin Drug Discov Devel 13:203-13.

French BA, Mazur W, Geske RS, Bolli R. 1994. Direct in vivo gene transfer into porcine myocardium using replication-deficient adenoviral vectors. Circulation 90:2414-24.

Fromes Y, Salmon A, Wang X, et al. 1999. Gene delivery to the myocardium by intrapericardial injection. Gene Ther 6:683-8.

Gaffney MM, Hynes SO, Barry F, O'Brien T. 2007. Cardiovascular gene therapy: current status and therapeutic potential. Br J Pharmacol $152: 175-88$

Giacca M, Zacchigna S. 2012. Virus-mediated gene delivery for human gene therapy. J Control Release 161:377-88.

Giordano FJ. 2003. Retrograde coronary perfusion: a superior route to deliver therapeutics to the heart? J Am Coll Cardiol 42:1129-31.

Guzman RJ, Lemarchand P, Crystal RG, Epstein SE, Finkel T. 1993. Efficient gene transfer into myocardium by direct injection of adenovirus vectors. Circ Res 73:1202-7.

Hajjar RJ, Schmidt U, Matsui T, et al. 1998. Modulation of ventricular function through gene transfer in vivo. Proc Natl Acad Sci U S A 95:5251-6.

Hayase M, Del Monte F, Kawase Y, et al. 2005. Catheter-based antegrade intracoronary viral gene delivery with coronary venous blockade. Am J Physiol Heart Circ Physiol 288:H2995-3000.

Hiltunen MO, Turunen MP, Turunen AM, et al. 2000. Biodistribution of adenoviral vector to nontarget tissues after local in vivo gene transfer to arterial wall using intravascular and periadventitial gene delivery methods. Faseb j 14:2230-6.

Hoshino K, Kimura T, De Grand AM, et al. 2006. Three catheter-based 
strategies for cardiac delivery of therapeutic gelatin microspheres. Gene Ther 13:1320-7.

Hou J, Kang YJ. 2012. Regression of pathological cardiac hypertrophy: signaling pathways and therapeutic targets. Pharmacol Ther 135:337-54.

Kaplitt MG, Xiao X, Samulski RJ, et al. 1996. Long-term gene transfer in porcine myocardium after coronary infusion of an adeno-associated virus vector. Ann Thorac Surg 62:1669-76.

Katz MG, Fargnoli AS, Bridges CR. 2013a. Myocardial gene transfer: routes and devices for regulation of transgene expression by modulation of cellular permeability. Hum Gene Ther 24:375-92.

Katz MG, Fargnoli AS, Kendle AP, Bridges CR. 2017. Molecular Cardiac Surgery with Recirculating Delivery (MCARD): Procedure and Vector Transfer. Methods Mol Biol 1521:271-289.

Katz MG, Fargnoli AS, Pritchette LA, Bridges CR. 2012a. Gene delivery technologies for cardiac applications. Gene Ther 19:659-69.

Katz MG, Fargnoli AS, Swain JD, et al. 2012b. AAV6-betaARKct gene delivery mediated by molecular cardiac surgery with recirculating delivery (MCARD) in sheep results in robust gene expression and increased adrenergic reserve. J Thorac Cardiovasc Surg 143:720-726.e3.

Katz MG, Fargnoli AS, Williams RD, Bridges CR. 2013b. Gene therapy delivery systems for enhancing viral and nonviral vectors for cardiac diseases: current concepts and future applications. Hum Gene Ther 24:914-27.

Katz MG, Fargnoli AS, Williams RD, Bridges CR. 2014a. The road ahead: working towards effective clinical translation of myocardial gene therapies. Ther Deliv 5:39-51.

Katz MG, Fargnoli AS, Williams RD, Bridges CR. 2014b. Surgical methods for cardiac gene transfer. Future Cardiol 10:323-6.

Katz MG, Fargnoli AS, Williams RD, et al. 2014c. Safety and efficacy of high-dose adeno-associated virus 9 encoding sarcoplasmic reticulum $\mathrm{Ca}(2+)$ adenosine triphosphatase delivered by molecular cardiac surgery with recirculating delivery in ovine ischemic cardiomyopathy. J Thorac Cardiovasc Surg 148:1065-72, 1073e1-2; discussion1072-3.

Katz MG, Swain JD, Fargnoli AS, Bridges CR. 2010. Gene therapy during cardiac surgery: role of surgical technique to minimize collateral organ gene expression. Interact Cardiovasc Thorac Surg 11:727-31.

Katz MG, Swain JD, Tomasulo CE, et al. 2011. Current strategies for myocardial gene delivery. J Mol Cell Cardiol 50:766-76.

Kawase Y, Ladage D, Hajjar RJ. 2011. Rescuing the failing heart by targeted gene transfer. J Am Coll Cardiol 57:1169-80.

Kawase Y, Ly HQ, Prunier F, et al. 2008. Reversal of cardiac dysfunction after long-term expression of SERCA2 a by gene transfer in a pre-clinical model of heart failure. J Am Coll Cardiol 51:112-9.

Kaye DM, Preovolos A, Marshall T, et al. 2007. Percutaneous cardiac recirculation-mediated gene transfer of an inhibitory phospholamban peptide reverses advanced heart failure in large animals. J Am Coll Cardiol 50:253-60.

Kornowski R, Fuchs S, Leon MB, Epstein SE. 2000. Delivery strategies to achieve therapeutic myocardial angiogenesis. Circulation 101:454-8.

Kupatt C, Wichels R, Deiss M, et al. 2002. Retroinfusion of NFkappaB decoy oligonucleotide extends cardioprotection achieved by CD18 inhibition in a preclinical study of myocardial ischemia and retroinfusion in pigs. Gene Ther 9:518-26.

Ladage D, Ishikawa K, Tilemann L, Muller-Ehmsen J, Kawase Y. 2012. Percutaneous methods of vector delivery in preclinical models. Gene Ther 19:637-41.

Lamping KG, Rios CD, Chun JA, et al. 1997. Intrapericardial administration of adenovirus for gene transfer. Am J Physiol 272:H310-7.

Lassaletta AD, Chu LM, Sellke FW. 2011. Therapeutic neovascularization for coronary disease: current state and future prospects. Basic Res

\section{Cardiol 106:897-909.}

Lazarous DF, Shou M, Stiber JA, et al. 1997. Pharmacodynamics of basic fibroblast growth factor: route of administration determines myocardial and systemic distribution. Cardiovasc Res 36:78-85.

Lin H, Parmacek MS, Morle G, Bolling S, Leiden JM. 1990. Expression of recombinant genes in myocardium in vivo after direct injection of DNA. Circulation 82:2217-21.

McMahon JM, Wells DJ. 2004. Electroporation for gene transfer to skeletal muscles: current status. BioDrugs 18:155-65.

Melo LG, Pachori AS, Gnecchi M, Dzau VJ. 2005. Genetic therapies for cardiovascular diseases. Trends Mol Med 11:240-50.

Packer M, Coats AJ, Fowler MB, et al. 2001. Effect of carvedilol on survival in severe chronic heart failure. N Engl J Med 344:1651-8.

Quarck R, Holvoet P. 2004. Gene therapy approaches for cardiovascular diseases. Curr Gene Ther 4:207-23.

Raake P, von Degenfeld G, Hinkel R, et al. 2004. Myocardial gene transfer by selective pressure-regulated retroinfusion of coronary veins: comparison with surgical and percutaneous intramyocardial gene delivery. J Am Coll Cardiol 44:1124-9.

Raake PW, Hinkel R, Muller S, et al. 2008. Cardio-specific long-term gene expression in a porcine model after selective pressure-regulated retroinfusion of adeno-associated viral (AAV) vectors. Gene Ther 15:12-7.

Rengo G, Lymperopoulos A, Leosco D, Koch WJ. 2011. GRK2 as a novel gene therapy target in heart failure. J Mol Cell Cardiol 50:785-92.

Rengo G, Lymperopoulos A, Zincarelli C, et al. 2009. Myocardial adenoassociated virus serotype 6-betaARKct gene therapy improves cardiac function and normalizes the neurohormonal axis in chronic heart failure. Circulation 119:89-98.

Roques C, Salmon A, Fiszman MY, Fattal E, Fromes Y. 2007. Intrapericardial administration of novel DNA formulations based on thermosensitive Poloxamer 407 gel. Int J Pharm 331:220-3.

Rose EA, Gelijns AC, Moskowitz AJ, et al. 2001. Long-term use of a left ventricular assist device for end-stage heart failure. N Engl J Med 345:1435-43.

Schwarz ER, Speakman MT, Patterson M, et al. 2000. Evaluation of the effects of intramyocardial injection of DNA expressing vascular endothelial growth factor (VEGF) in a myocardial infarction model in the rat--angiogenesis and angioma formation. J Am Coll Cardiol 35:1323-30.

Scott NA, Cipolla GD, Ross CE, et al. 1996. Identification of a potential role for the adventitia in vascular lesion formation after balloon overstretch injury of porcine coronary arteries. Circulation 93:2178-87.

Sharif F, Daly K, Crowley J, O’Brien T. 2004. Current status of catheterand stent-based gene therapy. Cardiovasc Res 64:208-16.

Sharif F, Hynes SO, McMahon J, et al. 2006. Gene-eluting stents: comparison of adenoviral and adeno- associated viral gene delivery to the blood vessel wall in vivo. Hum Gene Ther 17:741-50.

von Degenfeld G, Raake P, Kupatt C, et al. 2003. Selective pressureregulated retroinfusion of fibroblast growth factor-2 into the coronary vein enhances regional myocardial blood flow and function in pigs with chronic myocardial ischemia. J Am Coll Cardiol 42:1120-8.

Wever-Pinzon O, Stehlik J, Kfoury AG, et al. 2012. Ventricular assist devices: pharmacological aspects of a mechanical therapy. Pharmacol Ther 134:189-99.

White JD, Thesier DM, Swain JB, et al. 2011. Myocardial gene delivery using molecular cardiac surgery with recombinant adeno-associated virus vectors in vivo. Gene Ther 18:546-52.

Wright MJ, Wightman LM, Latchman DS, Marber MS. 2001. In vivo myocardial gene transfer: optimization and evaluation of intracoronary gene delivery in vivo. Gene Ther 8:1833-9. 